\title{
Core-mantle interactions resulting from sudden changes in the earth's moment of inertia
}

\author{
S. Pearce \\ School of Computing Science, Simon Fraser University, Canada
}

\begin{abstract}
The effect of sudden changes in the Earth's moment of inertia on the hydromagnetic state of the core is studied. Rapid changes in georotation, due to ice age transgression and regression, are described as varying boundary conditions in an axisymmetric Earth model containing both viscous and electromagnetic coupling. The deterministic equations describing the limit of rapid rotation are employed in conjunction with restricted 2-D predictive magneto-fluid equations. A kinematic description is adopted for both buoyancy driven mass motions and the regeneration of the poloidal magnetic field. A pseudo-spectral method is used to solve the incompressible magneto-fluid equations. The variables are collocated in radius using Chebyshev polynomials and the pseudospectral evaluations in colatitude are done using associated Legendre polynomials. Time dependence and magnetic diffusion are controlled by a modified second order semi-implicit Runge-Kutta scheme. Steady state boundary layers, arising from differential motion of the outer core boundaries, were found to induce significant departures for both $\alpha^{2}$ - and $\alpha \omega$-dynamo steady state configurations. In particular, the toroidal field is found to reverse completely in the $\alpha^{2}$ model. The hydromagnetic communication time of the core, determined by the predictive magneto-fluid equations supports these deterministic calculations. The actual lag-rate of the inner core has yet to be established. Within the context of this model, it is concluded that a causal connection is plausible between geomagnetic transients and significant changes in the Earth's moment of inertia.

Keywords: dynamo theory, geomagnetic reversal, eustacy, MHD, CMHD, spectral methods, spectral collocation.
\end{abstract}




\section{Introduction}

This paper is essentially a case-study in Computational Magnetohydrodynamics (CMHD) utilizing Spectral Methods. In particular, it is concerned with the geophysical problem of the hypothetical connection between sudden, significant eustatic change and the hydromagnetic state of Earth's deep interior [1-9]. This proposition may be outlined as follows: Significant sea-level changes have occurred many times throughout Earth's history as a consequence of transgressions and regressions of ice-ages, thus producing global sea-level changes that are typically on the order of 100 meters. Rapid sea-level rise will augment the mantle's moment of inertia if the eustatic sea-level rise is rapid compared to the viscous response time of the mantle, which is the case for ice-age regressions. In order to conserve angular momentum, the mantle will spin-down by one part in $10^{5}$ amounting to a loss of one second per day.

If the time-scale of such a spin-down is rapid compared to the hydromagnetic response time of the outer core, which is arguably true, then this sort of event on the surface of the Earth may produce an impulse in Earth's deep interior which could initiate a geomagnetic transient (excursion or reversal). In what follows, we assume these geophysical mechanisms and present a spectral method-based Earth model to describe this evolution problem.

The numerical method utilized to approximate the solution space of the coupled set of PDEs is spectral collocation. Spectral methods are extremely accurate and, therefore, are the only numerical technique useful enough for evolution problems involving large temporal integration. Eventually, we will turn to the details of this calculation, but first we must characterize the physical problem at hand. What follows in the next two sections is a condensed discussion of the pertinent geophysics.

\subsection{The core environment}

The evolution of an astrophysical-scale magnetic field $\mathbf{B}$ embedded in an electrically conducting fluid of velocity $\mathbf{v}$ and electrical conductivity $\sigma$ is described by an linear inhomogeneous diffusion equation usually referred to as the hydromagnetic induction equation (viz., [9]),

$$
\frac{\partial \mathbf{B}}{\partial t}=\nabla \times(\mathbf{v} \times \mathbf{B})+\nabla \times(\eta \nabla \times \mathbf{B})
$$

where

$$
\eta=\frac{1}{\sigma \mu_{0}}
$$

and $\mu_{0}$ is the permeability of free space. If the fluid has uniform electrical conductivity the last term collapses to $\eta \nabla^{2} \mathbf{B}$, revealing $\eta$ as the magnetic diffusivity. This assumption is commonly made as little physical insight is gained from the additional computational expense incurred from a continuously variable conductivity model (ie., [10]). The momentum equation describing the evolution 
of the Earth's fluid outer core is

$$
\rho \frac{\partial \mathbf{v}}{\partial t}+(\mathbf{v} \cdot \nabla \mathbf{v})=-\nabla p+\sum \mathcal{F}_{i}
$$

where $\mathcal{F}_{C}=2 \boldsymbol{\Omega} \times \mathbf{v}$ is the Coriolis force, $\mathcal{F}_{L}=\mathbf{B} \cdot \nabla \mathbf{B} / \mu_{0}$ is the Lorentz force, $\mathcal{F}_{A}=\rho \mathbf{g}$ is the Archimedean force and $\mathcal{F}_{v}=\mu \nabla^{2} \mathbf{v}+\frac{\mu}{3} \nabla(\nabla \cdot \mathbf{v})$ is the viscous force. The outer core can be modeled as an incompressible fluid, which provides overwhelming simplifications.

Let us gauge the relative importance of the individual terms contained in the momentum equation within the context of the Earth's core. The low fluid velocities previously mentioned suggest that the inertial acceleration $(\mathbf{v} \cdot \nabla) \mathbf{v}$ is very small compared to the Coriolis acceleration $2 \Omega \times \mathbf{v}$ under steady state conditions. Estimation of the remaining viscous dissipation term is hindered by our poor understanding of the outer core viscosity - the literature values span nearly fourteen orders of magnitude (viz., [11]). However, values at the lower limit, $\nu \sim 10^{-6} \mathrm{~m}^{2} \cdot \mathrm{s}^{-1}$ appear most likely (i.e., [12]). Note that the kinematic viscosity is $\nu=\mu / \rho$. The viscous term is orders of magnitude smaller than the Coriolis term even if one assumes the largest estimates of $\nu$. Also note that the effective pressure is no longer a thermodynamic variable but rather a fluid parameter that insures the solenoidity of the fluid (viz., [13]).

From our crude estimations, equation (3) can be reduced to

$$
2 \rho \boldsymbol{\Omega} \times \mathbf{v}=-\nabla p+\rho \mathbf{g}+\frac{(\nabla \times \mathbf{B}) \times \mathbf{B}}{\mu_{0}}
$$

where balance exists between the Coriolis force, pressure gradients, the gravitational force and the Lorentz force. The observation that most astrophysical dipole fields are closely aligned with their rotation axes strongly supports the contention that the Coriolis force is dominant. Then with gravitational forcing, the specifics of outer core fluid motions determine how the remaining two forces balance with the Coriolis force. Equation (4) forms the basis for the well known limit of rapid rotation (i.e., [14]).

It was first noted by Elsasser [15] shear is very efficient in generating cosmic magnetic fields. The process whereby an azimuthal magnetic field is generated by differential rotation is termed the $\omega$-effect (viz., [16]). A meridional field, such as a dipole, cannot be produced in this manner. In fact, Cowling's theorem [17] rules out complete axisymmetry - there must be an element of asymmetry to have self sustained dynamo action. Parker [18] proposed meridional field regeneration through a mechanism whereby rising helical convective plumes distort the azimuthal component into the meridional plane. This is referred to as the $\alpha$-effect [19] and is discussed in more detail later. If the $\omega$-effect is weak the dynamo is referred to as of $\alpha^{2}$-type (geostrophic). Otherwise, it is referred to as an $\alpha \omega$-dynamo (magnetostrophic). 


\section{The earth model}

We assume a three-component view of Earth (inner core, outer core and mantle) we now assume axisymmetry. The calculation reduces to two dimensions with azimuthal independence in mean quantities. This approach has been developed in recent years, especially in mean-field magnetohydrodynamics [20]. Inherent in this assumption, however, is that radial convective motions possessing helicity, crucial to dynamo action, cannot be modeled explicitly. This poses no serious concerns for investigations of this kind, as shall be seen below.

The change in the core-mantle boundary conditions are that of uniform zonal shear. Furthermore, a change in the meridional velocity field is sought, which can be fully accounted for in this description. In spherical coordinates $(r, \theta, \phi)$ the large-scale structures of this model reside in the azimuthal plane $\phi$, and, accordingly, axisymmetry is justified. Let us now turn to the mathematical description of the core.

\subsection{MHD model equations}

The solenoidal decomposition of both field variables into poloidal and toroidal components allows one to rewrite the hydromagnetic induction equation in component form as

$$
\begin{aligned}
\frac{\partial A^{(i)}}{\partial t} & =\frac{\eta_{i}}{\eta_{o}} \nabla_{\phi}^{2} A^{(i)} \\
\frac{\partial B^{(i)}}{\partial t} & =\frac{\eta_{i}}{\eta_{o}} \nabla_{\phi}^{2} B^{(i)} \\
\frac{\partial A^{(o)}}{\partial t} & =\nabla_{\phi}^{2} A^{(o)}+\alpha B^{(o)}+\aleph_{2}\left(\psi, A^{(o)}\right) \\
\frac{\partial B^{(o)}}{\partial t} & =\nabla_{\phi}^{2} B^{(o)}+\aleph_{1}\left(v, A^{(o)}\right)-\aleph_{1}\left(B^{(o)}, \psi\right) \\
& +\alpha \hat{\mathbf{e}}_{\phi} \cdot \nabla \times\left[\xi \nabla \times\left(A^{(o)} \hat{\mathbf{e}}_{\phi}\right)\right]
\end{aligned}
$$

where

$$
\nabla_{\phi}^{2} \equiv\left(\nabla^{2}-\frac{1}{r^{2} \sin ^{2} \theta}\right)
$$

is the $\phi$-component of the spherical axisymmetric vector diffusion operator and the interaction terms, operating on some field quantities $\chi_{1}$ and $\chi_{2}$, are conveniently expressed as

$$
\aleph_{1}\left(\chi_{1}, \chi_{2}\right) \equiv \hat{\mathbf{e}}_{\phi} \cdot \nabla \times\left[\chi_{1} \hat{\mathbf{e}}_{\phi} \times \nabla \times\left(\chi_{2} \hat{\mathbf{e}}_{\phi}\right)\right]
$$

and

$$
\aleph_{2}\left(\chi_{1}, \chi_{2}\right) \equiv \hat{\mathbf{e}}_{\phi} \cdot\left[\nabla \times\left(\chi_{1} \hat{\mathbf{e}}_{\phi}\right) \times \nabla \times\left(\chi_{2} \hat{\mathbf{e}}_{\phi}\right)\right]
$$


(viz., [21,22]). The superscripts $(i)$ and $(o)$ refer to inner core and outer core quantities, respectively. The only relevant physics pertaining to the inner core is magnetic diffusion. We see that the $\alpha$-effect produces both poloidal and toroidal fields. Note that, for an $\alpha \omega$-dynamo, the $\alpha$-effect is overwhelmed by the $\omega$ effect, $\aleph_{1}\left(v, A^{(o)}\right)$. Also, poloidal field is generated by non-parallel interactions of both field quantities within meridional planes whereas both $\mathbf{v}_{T}-\mathbf{B}_{P}$ and $\mathbf{B}_{T}-\mathbf{v}_{P}$ interactions contribute to the toroidal field. Note also that axisymmetric motions alone cannot regenerate $A-$ this is Cowling's theorem.

Let us discuss the exact model prescription of the $\alpha$-effect. Any reasonable simplistic parameterization of the small-scale asymmetric flow will suffice in this endeavor. For example, Hollerbach and Jones [23, 24] found the simple expression $\alpha=\alpha_{0} \cos \theta$ an adequate description. The choice of $\alpha$ as a scalar amounts to all components of the tensor $\xi_{i j}$ vanishing except the diagonals; that is, $\xi_{11}=\xi_{22}=$ $\xi_{33}=\alpha$. Hollerbach and Jones [25] utilized a tensor description, with only $\xi_{33}$ non-vanishing, and found satisfactory results.

Let us now turn to the momentum equation. The vector potential formalism for the evolution of the poloidal magnetic field presents better stability characteristics for numerical simulations. In addition, the rotational form describing the fluid vorticity can be employed where, in a similar poloidal-toroidal decomposition, is advantageous in a computational sense (viz., Canuto et al. [26]). As such, the momentum equation may be expressed in the following streamfunction-vorticity formalism

$$
\begin{aligned}
\frac{\partial \omega}{\partial t} & =R o^{-1}\left\{2 v z-E \nabla_{\phi}^{4} \psi-\Lambda\left[\aleph_{1}\left(B^{(o)}, B^{(o)}\right)-\aleph_{1}\left(\nabla_{\phi}^{2} A^{(o)}, A^{(o)}\right)\right]+\frac{\partial F}{\partial \theta}\right\} \\
& +\aleph_{1}(v, v)-\aleph_{1}\left(\nabla_{\phi}^{2} \psi, \psi\right) \\
\frac{\partial v}{\partial t} & =R o^{-1}\left\{2 \psi z+E \nabla_{\phi}^{2} v+\Lambda \aleph_{2}\left(B^{(o)}, A^{(o)}\right)\right\}+\aleph_{2}(\psi, v)
\end{aligned}
$$

where $\omega$ is related to the stream function by

$$
\omega=-\nabla_{\phi}^{2} \psi
$$

and

$$
\frac{\partial}{\partial z} \equiv \cos \theta \frac{\partial}{\partial r}-\frac{\sin \theta}{r} \frac{\partial}{\partial \theta}
$$

(viz, $[21,22,26])$. The operators $\aleph_{1}$ and $\aleph_{2}$ were defined earlier by equations (10) and (11) and describe the nonlinear interactions. Note there is no need for the superscript notation for fluid quantities, which are restricted to the outer core. The pressure no longer appears explicitly when taking the curl of the meridional part of the momentum equation. 
Noting that $R o \sim 10^{-8}$ then the limit of rapid rotation is obtained by solving only the diagnostic set of equations

$$
\begin{gathered}
2 \frac{\partial v}{\partial z}-E \nabla_{\phi}^{4} \psi=\Lambda\left[\aleph_{1}\left(B^{(o)}, B^{(o)}\right)+\aleph_{1}\left(\nabla_{\phi}^{2} A^{(o)}, A^{(o)}\right)\right]+\frac{\partial F}{\partial \theta} \\
2 \frac{\partial}{\partial z}+E \nabla_{\phi}^{2} v=-\Lambda \aleph_{2}\left(B^{(o)}, A^{(o)}\right)
\end{gathered}
$$

contained in equations (12) and (13).

As a final point we note that, in the limit of rapid rotation, the neglect of inertia necessitates that the total torque must vanish on the inner core boundary [27]. There must, therefore, be torque balance. The simplest way to accomplish this is by assuming that electromagnetic and viscous torques compete at the boundary (i.e., [23]). Of course, the full momentum equations do not require this condition nor if the inner core is forced to rotate arbitrarily. This leads naturally into a full summary of all boundary conditions.

\subsection{Boundary conditions}

The matching conditions on the vector potential at the inner core boundary are due to the continuity of both $B_{r}$ and $B_{\theta}$. Recalling that $\mathbf{B}=\nabla \times \mathbf{A}$ then, across any radial boundary, we must have both matching of magnetic field and its derivative.

For an insulating mantle, $\mathbf{B}_{p}$ must match an external potential field. Then, integrating across the boundary, one has that

$$
\nabla_{\phi}^{2} A=0
$$

The matching and jump conditions on the toroidal magnetic field, at the coremantle boundary, are:

$$
\begin{aligned}
B^{(o)}\left(R_{o}, \theta, t\right) & =B^{(m)}\left(R_{o}, \theta, t\right) \\
\left.\eta_{o} \frac{1}{r} \frac{\partial\left(r B^{(o)}\right)}{\partial r}\right|_{r=R_{o}^{-}} & =\left.\eta_{m} \frac{1}{r} \frac{\partial\left(r B^{(m)}\right)}{\partial r}\right|_{r=R_{o}^{+}}
\end{aligned}
$$

Similar such conditions exist at the inner-outer core boundary, with $\eta_{i}=\eta_{o}$ in this calculation.

The boundary conditions associated with the fluid flow vary between the rapid rotation limit and fully predictive equations. In both cases, the no-slip condition for the stream function is

$$
\psi=\frac{\partial \psi}{\partial r}=0
$$

Notice that there are no physical boundary conditions for the vorticity in the streamfunction-vorticity formulation [26].

The toroidal velocity field must take into account the fact that the inner core does not necessarily co-rotate with the mantle. Also the no-slip boundary conditions on the flow in steady state at the base of the mantle must be enforced. Thus, we must 
have that

$$
\begin{aligned}
v=\Omega_{i} R_{i} \sin \theta, & r & =R_{i} \\
v=0, & r & =R_{o}
\end{aligned}
$$

where $\Omega_{i}$ is the solid body rotation rate of the inner core.

Clearly, the simple prescription

$$
v=\triangle \Omega_{m} R_{o} \sin \theta, \quad r=R_{o}
$$

enables us to incorporate the mantle as a boundary condition.

\section{The numerical method}

The model equations were solved pseudo-spectrally. A thorough review of all spectral methods was given by Canuto et al. [26]. The algorithmic approach, developed for the limit of rapid rotation, is structurally similar to Hollerbach [28]. Specific details can be found in Hollerbach and Jones [23] and Hollerbach [28]. However, both deterministic and fully predictive calculations employ a modified second order semi-implicit Runge Kutta scheme for temporal integration and for the evaluation of diffusive terms. All boundary conditions are applied here for the latter algorithm. All non-diffusive terms are solved pseudo-spectrally.

The magnetic field in the inner core is expanded as

$$
\begin{aligned}
& A^{(i)}\left(r_{j}^{(i)}, \theta_{i}, t\right)=\sum_{n=1}^{N_{1}} \sum_{m=1}^{M_{1}+2} \tilde{A}_{n m}^{(i)}(t) T_{m-1}\left(x_{j}^{(i)}\right) P_{2 n-1}^{1}\left(\cos \theta_{i}\right) \\
& B^{(i)}\left(r_{j}^{(i)}, \theta_{i}, t\right)=\sum_{n=1}^{N_{1}} \sum_{m=1}^{M_{1}+2} \tilde{B}_{n m}^{(i)}(t) x_{j}^{(i)} T_{m-1}\left(x_{j}^{(i)}\right) P_{2 n}^{1}\left(\cos \theta_{i}\right)
\end{aligned}
$$

where all tildes represent spectral coefficients. The subscripts $i$ and $j$ refer to the collocation points in angle and radius, respectively. The angular grid points are the $N_{2}$ zeros of $P_{2 N_{2}+1}^{1}$ on $(0,1)$, using a root finding algorithm, and the radial collocation points are the $M_{1} / 2$ zeros of $T_{M_{1}+1}\left(x^{(i)}\right)$ on $(0,1)$. The additional coefficients in the radial expansion relate to and are determined by the boundary conditions (as in the outer core expansions below). The radial domain of the Chebyshev polynomials is mapped according to $x_{j}^{(i)}=r_{j}^{(o)} / R_{i}$. Note the expansion in $B^{(i)}$ imposes the correct symmetry in radius. Also, the radial symmetries are related to the equatorial symmetries, and this condition is necessary for $A^{(i)}$ to be antisymmetric and for $B^{(i)}$ to be symmetric.

The outer core solution is expanded in a similar manner. Note that there is no time dependence in the fluid quantities in the limit of rapid rotation. Also the number of collocation points differs between fluid and magnetic field calculations. 
This is to resolve fluid boundary layers and, also, for proper de-aliasing (viz., [26]). The collocation grid in radius is established from the $M_{1}$ zeros of $T_{M_{1}}\left(x_{j}^{(o)}\right)$ on $(-1,1)$. The domain of the Chebyshev polynomials is mapped according to

$$
r_{j}^{(o)}=\frac{R_{o}+R_{i}}{2}+\frac{R_{o}-R_{i}}{2} x_{j}^{(o)}
$$

Accordingly, all radial derivatives are evaluated, in general, as

$$
\frac{d^{p} T_{m}}{d r^{p}}=\frac{d^{p} T_{m}}{d x^{p}}\left(\frac{d x}{d r}\right)^{p}
$$

We assume $R_{i}=1 / 2, R_{o}=3 / 2$. Similar rules apply to the inner core.

The boundary conditions regarding fluid flow are now described in specific detail. For the limit of rapid rotation we can cast equation (23) as

$$
\sum_{m=1}^{M_{2}+4} \tilde{\psi}_{n m}(-1)^{m}=\sum_{m=1}^{M_{2}+4} \tilde{\psi}_{n m}(-1)^{m}(m-1)^{2}=0
$$

at the inner core boundary and

$$
\sum_{m=1}^{M_{2}+4} \tilde{\psi}_{n m}=\sum_{m=1}^{M_{2}+4} \tilde{\psi}_{n m}(m-1)^{2}=0
$$

at the outer core boundary. The fully predictive relation is clearly similar.

Since $P_{1}^{1}(\cos \theta)=-\sin \theta$, equation (24) becomes the pair

$$
\sum_{m=1}^{M_{2}+2} \tilde{v}_{1 m}(-1)^{m}=R_{i} \Omega_{i}
$$

and

$$
\sum_{m=1}^{M_{2}+2} \tilde{v}_{n m}(-1)^{m}=0, \quad n>1
$$

where the first determines $\Omega_{i}$, and the second gives the boundary condition for $n>1 . \Omega_{i}$ is obtained from the evaluation of the instantaneous magnetic torque on the inner core.

The toroidal velocity field vanishes at the outer core boundary in the case of no mantle spin-up. Thus,

$$
\sum_{m=1}^{M_{2}^{\prime}+2} \tilde{v}_{n m}=0 \quad \forall n
$$

However, for mantle spin-up we must consider equation (23) which, upon expansion, has the form

$$
\tilde{v}_{n}=-R_{o} \triangle \Omega_{m} \delta_{1, n}
$$


which, as with the inner core analysis above, becomes

$$
\sum_{m=1}^{M_{2}^{\prime}+2} \tilde{v}_{1 m}=R_{o} \triangle \Omega_{m} .
$$

remembering that $\Omega_{m}$ is the mantle's angular velocity (there is no summing index implied). Similar expansions exist for the magnetic field.

\section{Model calculations}

We are interested in the effect of significant zonal shear at the core-mantle boundary. We impose a linearly increasing rate of differential rotation on the steady state $\alpha^{2}$-dynamo. Differential rotation increased linearly over some 30 $\mathrm{ka}$, the timescale of the Late Pleistocene eustatic sea-level rise, reaching a final magnitude comparable to the largest toroidal flows found in the steady state $\alpha^{2}$ dynamo calculations (one hundred times less than that of westward drift).

The $\alpha^{2}$-dynamo is now actually an $\alpha \omega$-dynamo with a differing, relatively weak, $\omega$-effect from those computed without differentially rotating boundaries. Torque balance in the steady state $\alpha^{2}$-dynamo produced greater inner core rotation than is allowed in the present calculation. Accordingly, there is no noteworthy departure from dynamo action [23]. However, if the final differential rotation rate is raised to be comparable to westward drift the results become striking. Of tantamount interest is the reversal of the magnetic field. This event is expanded in Figure 1 for the toroidal magnetic field. Each panel is at intervals of 6,000 years. Notice that the inner core appears to help facilitate the reversal. Also observe the migration of field structures toward the equator, where the toroidal velocity is largest.

The results presented in the previous section would be more reliable if it could be demonstrated that the timescale of spin-up is more rapid than $d t$ for each respective calculation. This elicits a simple calculation regarding the transient inertial effects neglected thus far. This returns the discussion to the central issue of the communication time of the outer core describable from the fully predictive model (14) and (15).

A simple calculation was performed in order to reduce the complexity of the problem by imagining the outer core as a set of rigid concentric shells. This effectively reduces the mechanics to one dimension. With this simple description the Alfvén crossing time of the outer core can be estimated. $R o \sim 10^{-8}$ is consistent with the parameters used above and, accordingly, the time step must be some $10^{4}$ times smaller than in all previous calculations. Unfortunately, the algorithm did not allow calculations to proceed with $R o=E<10^{-6}$, similar to numerical barrier encountered by others (i.e., [29-30]) using spectral methods. Note that the production of toroidal magnetic field propagates to the inner core boundary at roughly the $3 / 4$ point in this calculation, which spans some 120 years. Other runs, for larger $R o$, suggest a linear relationship between $R o$ and $d t$ as displayed by the evolution equation for $v$ above. This amounts to nothing more than linearly extrapolating the results calculated for effectively smaller core 

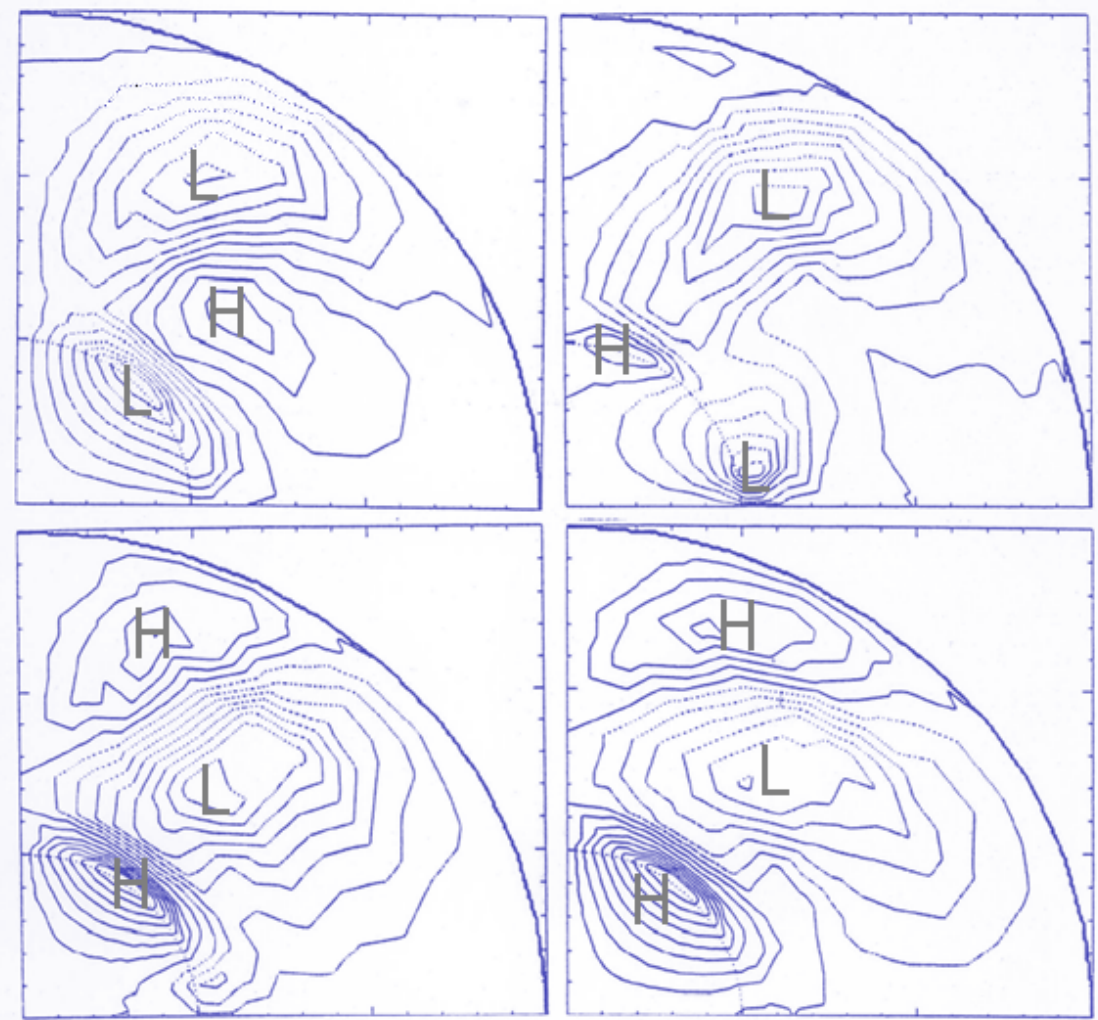

Figure 1: Reversal of the toroidal magnetic field.

geometries (embodied in $R o \propto 1 / L^{2}$ ). Thus, with $R o \sim 10^{-8}$, we expect a core communication time of less than a year or so, supporting the use of the limit of rapid rotation within this context.

\section{Discussion and conclusions}

The current results differ from previous calculations [28] who explored the regime of higher Reynolds numbers (and a slightly lower Elsasser number) as compared to this study. Moreover, this calculation suggests that the formation of complicated Stewartson layers is not expected in the outer core. In addition, the Ekman layers are confined within the cylinder tangent to the inner core and parallel to the rotation axis.

Unlike earlier studies, in this investigation we have been concerned with timeevolving boundary conditions. Because the non-inertial calculations are inherently time-independent regarding the velocity field, our approach represents some 
aspects of the variation as a sequence of steady states. This aspect requires some justification.

In the previous section, the final result was an inertial calculation employed to lend support for the limit of rapid rotation during spin-up. Recall that the smaller $R o$ the faster the core communication time. Our employment of an unrealistically large Rossby number, for numerical tractability, can be interpreted as modeling a very small "Earth" $-R_{o} \propto 1 / \Omega L^{2}$. It was shown that such calculations could be linearly extrapolated, beyond our numerical limitations, into the regime expected for the Earth. For our model $R_{0} \sim 10^{-8}$ suggesting that information regarding changes in $\Delta \Omega_{m}$ are communicated throughout the entire core on a timescale of a year. This is on the order of the smallest time step utilized in this calculation lending support for the use of the deterministic momentum equation. Also, a constant relative rate of differential rotation, expected to first order during ice age regression, is a steady state core configuration. A principal issue of concern is the actual magnitude of inner core lag anticipated during transient ice age events. To pose it in another way; How fast does the inner core respond to electromagnetic torques? The moment of inertia of the inner core is roughly $5 \times 10^{34} \mathrm{~kg} \cdot \mathrm{m}^{2}$. Changing its angular velocity by one part in $10^{-5}$ over some $10^{3}$ years requires roughly $10^{15} \mathrm{~N} \cdot \mathrm{m}$. Evaluation of the net electromagnetic torque available at the inner core is difficult as our quasi-steady state solution sequences suggest rapid expulsion of $B^{(i)}$. The transient build-up of toroidal magnetic field largely determines this torque, and is anticipated to be quite high. Thus, differential rotation is not expected to be very large. Tentative calculations, including inner core inertia, support this view. However, definitive conclusions await a more complete analysis.

When considering these solutions, one must pay special attention to $A-$ it is the only component directly observable from the surface of the Earth. The steady state calculations of magnetic boundary layer structure, arising from differential outer core boundary motion, changed significantly with the addition, kinematically, of the $\alpha$-effect and buoyant force. Recall that the original steady state results are greatly affected by the presence of the electrically conducting inner core. Toroidal magnetic field is rapidly expelled from the inner core as a natural edifice for satisfying Taylor's constraint. This was true of both $\alpha^{2}$ - and $\alpha \omega$-dynamos [24].

The $\alpha^{2}$-dynamo appeared to be more stable during spin-up than the $\alpha \omega$-dynamo - there were no disordered solutions. However, the magnetic field was observed to reverse for a differential rotation of mere tenths of a millimeter per second. The sense of the differential rotation produced reversed $B$ which eventually dominated the solution. The inner core greatly facilitated this reversal. Strong Hartmann currents propagating equatorward allowed for the rapid expulsion of the transient $B^{(i)}$ near the equator. It was annihilated at the equator by its odd half from the other hemisphere. Note that the time scale for the reversal was some $10 \mathrm{ka}-$ consistent with the paleomagnetic evidence.

In the context of our model calculations the following tentative conclusions can be drawn. Differential rotation of the outer core boundaries is capable of inducing significant meridional circulation patterns that, when entering regions of dynamo 
activity, promote violations of Taylor's constraint and distort the magnetic field structure, causing disruption of dynamo action. The greater the rate of relative differential rotation the more pronounced this effect. The point at which the $\alpha \omega$ dynamo solutions become disordered is when the overall shear is comparable to the westward drift velocity. However, we expect the rate of differential rotation of the outer core boundaries to remain well below this value given reasonable timescale ( $\sim 10^{3}$ years) for changes in the angular velocity of the mantle. Within the context of these calculations, however, significant departures of both $\alpha^{2}$ - and $\alpha \omega$-dynamo solutions are observed - especially when considering the evolution of $A$. This suggests that the regressive episodes of ice age events can trigger departures of steady state geodynamo activity.

\section{References}

[1] Olausson, E. and Svenonius, B. The relation between glacial ages and terrestrial magnetism. Boreas, 2:109-115, 1973.

[2] Doake, C. S. M. A possible effect of ice ages on the Earth's magnetic field, Nature 267:415, 1977.

[3] Doake, C. S. M. Climatic change and geomagnetic field reversals: a statistical correlation, Earth Planet. Sci. Lett., 38:313, 1978.

[4] Pal, P. C. and Creer, K. M. Geomagnetic reversal supurs and episodes of extraterrestrail catastrophism, Nature, 320:148-150, 1986.

[5] Muller, R. A. and Morris, D. E. Geomagnetic reversals from impacts on the Earth. Geophys. Res. Lett., 13:1177-1180m, 1986.

[6] Burek, P. J. and Wänke, H. Impacts and glacio-eustasy, plate-tectonic episodes, geomagnetic reversals; A concept to facilitate detection of impact events, Phys. Earth Plan. Int., 50:183-194, 1988.

[7] Pal, P. C. Rotational and geomagnetic changes during the last glaciation period. In: New Approaches in Geomagnetism and the Earth's Rotation, ed, Flodmark, S., World Scientific Pub. Co, 1991.

[8] Valentin Krassilov, Sophia Barinova. Sea Level - Geomagnetic Polarity Correlation as Consequence of Rotation Geodynamics. Earth Science, Vol. 2, No. 1, 2013, pp. 1-8. doi: 10.11648/j.earth.20130201.11.

[9] Parker, E. N. Cosmical Magnetic Fields, Their Origin and Their Activity (Oxford: Clarendon), 1979.

[10] Levy, E. H. and Pearce, S. J. Steady state toroidal magnetic field at Earth's core mantle boundary. J. Geophys. Res., 96:3935-3942, 1991.

[11] Jacobs, J. A. The Earth's Core. Academic Press, 1987.

[12] Gans, R. F. Viscosity of the Earth' core, J. Geophys. Res., 77:360, 1972.

[13] Gresho, P. M. Incompressible fluid dynamics: Some fundamental formulation issues, Ann. Rev. Fluid Mech., 23:413-453, 1991.

[14] Fearn, D. R., Roberts, P. H. and Soward, A. M. Convection, stability and the dynamo. In: Energy, Stability and Convection, eds G. P. Galdi and B. Straughan, Pitman Research Notes in Mathematics series, Longmans, 168:60-324, 1988. 
[15] Elsasser, W. M. Induction effects in terrestrial magnetism III. Electric modes. Phys. Rev., 72:821-833, 1947.

[16] Levy, E. H. Generation of planetary magnetic fields, Ann. Rev. Earth Planet. Sci., 4:159-185, 1976.

[17] Cowling, T. G. The magnetic field of sunspots. Mon. Not. R. Astr. Soc., 94:39-48, 1934.

[18] Parker, E. N. Hydromagnetic dynamo models. Ap. J., 122:293, 1955.

[19] Steenbeck, M. and Krause, F. The generation of stellar and planetary magnetic fields by turbulent dynamo action. Z. Naturforsch, 219:1285-1296, 1966.

[20] Krause, F. and Rädler, K. -H. Mean-Field Magnetohydrodynamics and Dynamo Theory. Pergamon, Oxford, 1980.

[21] Malkus, W. V. R. and Proctor, M. R. E. The macrodynamics of $\alpha$-effect dynamos in rotating fluid systems, J. Fluid Mech., 67:417-444, 1975.

[22] Proctor, M. R. E. Numerical solutions of the nonlinear $\alpha$-effect dynamo equations, J. Fluid Mech., 80:769-784, 1977.

[23] Hollerbach, R. and Jones, C. A. A geodynamo model incorporating a finitely conducting inner core. Phys. Earth Plan. Int., 75:317-327, 1993.

[24] Hollerbach, R. and Jones, C. A. Influence of the Earth's inner core on geomagnetic fluctuations and reversals. Nature, 365:541-543. 1993.

[25] Hollerbach, R. and Jones, C. A. On the magnetically stabilizing role of the Earth's inner core. Phys. Earth Plan. Int., 365:541-543. 1995.

[26] Canuto, C., Hussaini, M. Y., Quarteroni, A. and Zang, T. A. Spectral Methods in Fluid Dynamics, Springer Verlag, 1989.

[27] Gubbins, D. Rotation of the inner core. J. Geophys. Res., B86:11695-11699, 1981.

[28] Hollerbach, R. Magnetohydrodyamic Ekman and Stewartson layers in a rotating spherical shell. Proc. R. Soc. Lond. A, 444:333-346, 1994.

[29] Zhang, K. A study of buoyancy-driven flows and magnetic field generation in rotating spherical shells. Ph.D. Thesis in Geophysics and Space Physics, UCLA, 1987.

[30] Glatzmaier, G. A. and Roberts, P. H. A three-dimensional self-consistent computer simulation of a geomagnetic field reversal, Nature, 377:203-209, 1995. 